\title{
Development of Environmentally - Friendly and Energy Efficient Refrigerant for Medium Temperature Refrigeration Systems
}

\author{
Piyanut Saengsikhiao ${ }^{1}$, Juntakan Taweekun ${ }^{2,}$, Kittinan Maliwan ${ }^{2}$, Somchai Sae-ung ${ }^{2}$, \\ Thanansak Theppaya ${ }^{2}$ \\ Energy Technology Program, Faculty of Engineering, Prince of Songkla University, Hat Yai, Songkhla 90112, Thailand \\ Department of Mechanical Engineering, Faculty of Engineering, Prince of Songkla University, Hat Yai, Songkhla 90112, Thailand
}

\section{ABSTRACT}

This research presents the development of environmentally-friendly and energy efficient refrigerant for medium temperature refrigeration systems that new azeotropic refrigerant mixture of hydrofluorocarbons and hydrocarbon that can retrofit in the refrigeration system using R404A. The medium back pressure refrigeration testing standard that follow CAN/ANSI/AHRI540 standard air-conditioning, heating, and refrigeration institute (AHRI) and The properties of refrigerants and refrigeration simulation system that used national institute of standards and technology (NIST) reference fluid thermodynamic and transport properties database (REFPROP) software and NIST vapor compression cycle model accounting for refrigerant thermodynamic and transport properties (CYCLE_D-HX) software. The methodology use decision tree function in datamining by rapid minor software that first of KDnuggets annual software poll that showed new azeotropic refrigerant mixture had cooling capacity, refrigerant effect, GWP and boiling point were lower than R404A but work and pressure for medium temperature refrigeration system of azeotropic refrigerant mixture were higher than R404A. The artificial intelligence (Al) by data mining technic can predictive environmentally-friendly and energy efficient refrigerant for medium temperature refrigeration. The result of refrigerant mixed by R134A, R32, R125 and R1270 and is consistent with the evolution of fourth-generation refrigerants that contain a mixture of HFCs and HCs which are required to produce a low-GWP, zero-ozone-depletionpotential (ODP), high-capacity, low-operating-pressure, and nontoxic refrigerant.

\section{Keywords:}

Refrigerant; refrigeration system; energy efficiency; environmentally friendly; data mining

Copyright @ 2020 PENERBIT AKADEMIA BARU - All rights reserved

\section{Introduction}

Energy use in Thailand's business sector is ranked second among overall energy users in the country, and is thus being targeted for energy-saving options [1]. The number of convenience stores in Thailand numbered to more than 20,000 locations in 2019, and this continuously increases on an annual basis [2]. The majority are open 24 hours per day, so the retail sector is the fourth largest

\footnotetext{
* Corresponding author.

E-mail address: juntakan.t@psu.ac.th

https://doi.org/10.37934/arms.71.1.1231
} 
consumer of energy in the business sector, consuming more energy than residences do [3]. The components that contribute to energy consumption of convenience stores in Thailand, ranked from highest to lowest, are refrigeration systems, air-conditioning systems, electrical equipment, and lighting [4,5]. However, proportions of energy use in convenience stores in Taiwan were previously ranked as shown in Figure 1 below [6]. The best options for reducing energy consumption in convenience stores in Thailand are high energy efficiency and an efficient energy-management system. A good example of energy savings in refrigeration systems is shown in Figure 2 below [7]. Energy savings in refrigeration systems can be achieved through decreased power consumption of the compressor, as this is the component that utilizes the most energy.

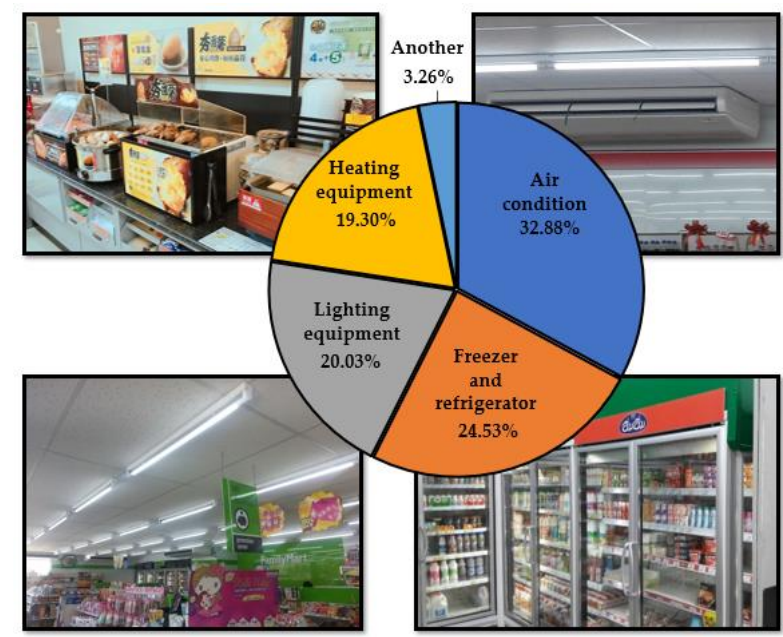

Fig. 1. Proportions of energy use in Taiwanese convenience stores [6]

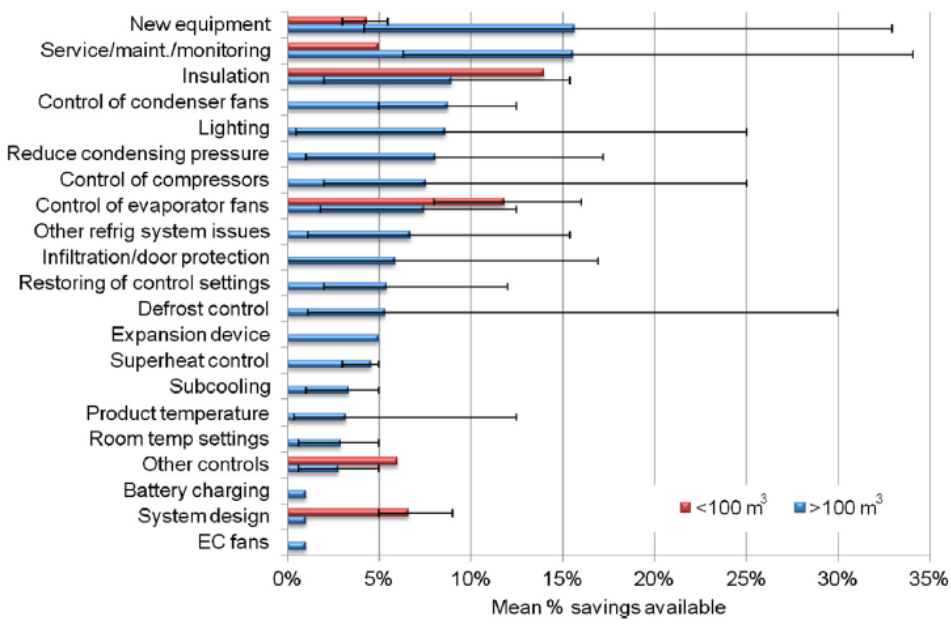

Fig. 2. Examples of energy savings in refrigeration systems [7]

Refrigerant trends in Thailand have shown improvements in increasing energy efficiency and decreasing global-warming potential (GWP), as shown in Figure $3[8,9]$, which is related to the hydrofluorocarbon (HFC) phase-down schedule, as shown in Figure 4 [10]. First- and secondgeneration refrigerants were composed of natural refrigerants and hydrocarbons (HCs), both of which do not impact the environment, have low GWP, and zero ozone-depletion potential (ODP) [1113]. R744 operates under high pressure, and is highly toxic and flammable [14-16]. Following the 
second generation, third-generation refrigerants were composed of chlorofluorocarbons (CFCs) [1719] and hydrochlorofluorocarbon (HCFCs) [20-22], which are easy to use, can operate under low pressure, and are nontoxic. However, they have high GWP and ODP, contributing to ozone depletion and global warming. Therefore, the development of refrigerants has significantly decreased ODP and GWP. Moreover, third-generation refrigerants, CFCs and HCHCs, were further developed into hydrofluorocarbon (HFC) refrigerants that still possessed low GWP and zero-ODP [23-25]. Fourthgeneration refrigerants are mainly hydrofluoroolefins (HFOs) with low GWP and low capacity [2628]. Therefore, they are refrigerants that are mixed with HFCs [29-31], HFOs [32-34], and HCs [3537]. Natural refrigerants are low-GWP, zero-ODP, high-capacity, low-pressure, and nontoxic [38-40].

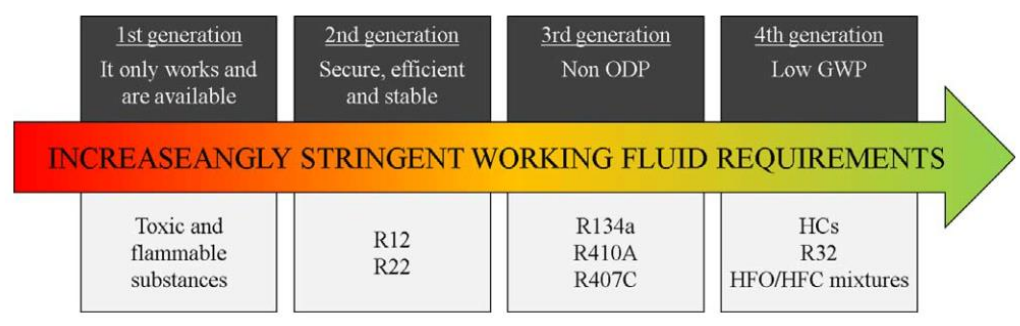

Fig. 3. Evolution of refrigerants $[8,9]$

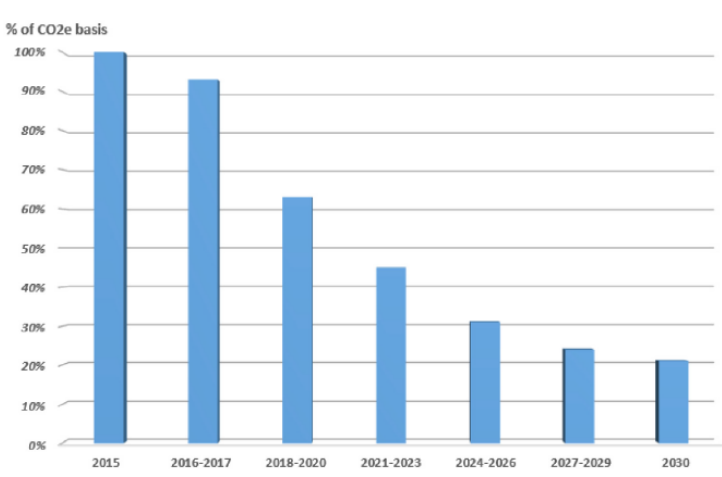

Fig. 4. Hydrofluorocarbon (HFC) phase-down schedule (Co2e \%) [10]

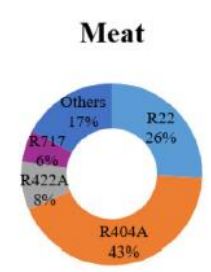

Fish

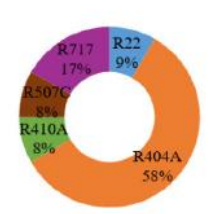

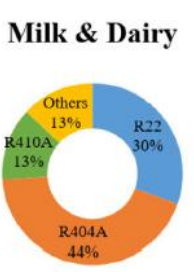

Wines

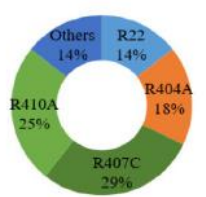

Fruit \& Vegetables

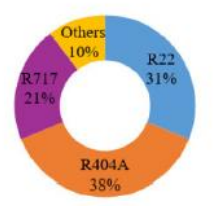

Food Distribution

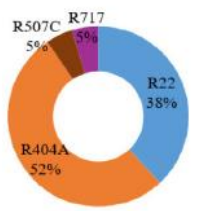

Fig. 5. Top refrigerants in food industry [9]

Refrigerants need to be low-GWP, zero-ODP, high-capacity, low-pressure, and nontoxic, and should thus be mixed with HCs and HFOs; however, current refrigerants are still highly flammable 
and have low capacity. An alternative is to incorporate other HFCs. R32 is low-GWP, zero-ODP, highcapacity, and nontoxic, but operates under high pressure and is not flammable, which is in contrast to R134A, which possesses highly similar properties but can operate under low pressure and has low capacity. Systems that operate with R22 [41], R417A [42], R417B [43], R422A [44], R422B [45], R422C [46], R422D [47], R424A [48], R433A [49], R437A [50], R438A [51] and R453A [52], were developed as an alternative to R22 and mixed with HCs and HFCs, as shown in Tables 1-4. Systems that operate with R134A [53], R450A [54], R456A [40], R513A [55], and R515A [40] were developed as an alternative to R134A, and mixed with HCs, HFCs, and HFOs, as shown in Table 5 . The fourthgeneration R404A was the basis for this research, and it is currently the most used refrigerant, as shown in Figure 5. R404A is an azeotropic blend of $143 a / 125 / 134$ a with zero ODP, and is nonflammable, nontoxic, and operates under low pressure, but has a GWP of 3922 [56]. R407A [57], R407F [58], R407H [59], R410A [60], R442A [52], R448A [61], R449A [62], R452A [63], R453A [64], and R463A [65] were developed to be retrofitted to replace R404A as shown in Table 6-9, and are mixed with HCs, HFOs, R134A, and R32. This is similar to the refrigerant that was developed for R22 and R134A but does not include R463A, which is being presented as a refrigerant for comparative purposes in this research as it is composed of $R 744$ (carbon dioxide), which is a natural refrigerant similar to R445A [64] and R455A [65]. These conform to the refrigerant-development trend and are an alternate option that can be mixed with HFC. The refrigerant proportion that was mixed with R125 was more or less similar to that of the R32 mixture, and it also possesses Class A1 nonflammability property.

Table 1

Properties of R22, R417A and R417B

\begin{tabular}{|c|c|c|c|c|c|c|c|}
\hline Condition & LT & MT & HT & LT & LT & MT & HT \\
\hline Refrigerant & & R22 [41] & & & & R417B [43] & \\
\hline $\begin{array}{l}\text { Composition } \\
\text { Mass }\end{array}$ & & R22 & & & & R125/134a/600 & \\
\hline $\begin{array}{l}\text { percentage } \\
\text { Boiling point }\end{array}$ & & 100 & & & & $79 / 18.3 / 2.7$ & \\
\hline $\begin{array}{c}\left({ }^{\circ} \mathrm{C}\right) \\
\text { Critical Pressure }\end{array}$ & & -40.80 & & & & -45.20 & \\
\hline $\begin{array}{c}\text { (kPa) } \\
\text { Critical }\end{array}$ & & 4,990 & & & & 3,737 & \\
\hline $\begin{array}{c}\text { Temperature } \\
\left({ }^{\circ} \mathrm{C}\right)\end{array}$ & & 96 & & & & 74 & \\
\hline ODP & & 0.055 & & & & 0 & \\
\hline GWP & & 1,600 & & & & 3,027 & \\
\hline Class & & $\mathrm{A} 1$ & & & & A1 & \\
\hline Lubricant type & & MO & & & & $\mathrm{MO} / \mathrm{POE}$ & \\
\hline
\end{tabular}




\section{Table 2}

Properties of R422A, R422B and R422C

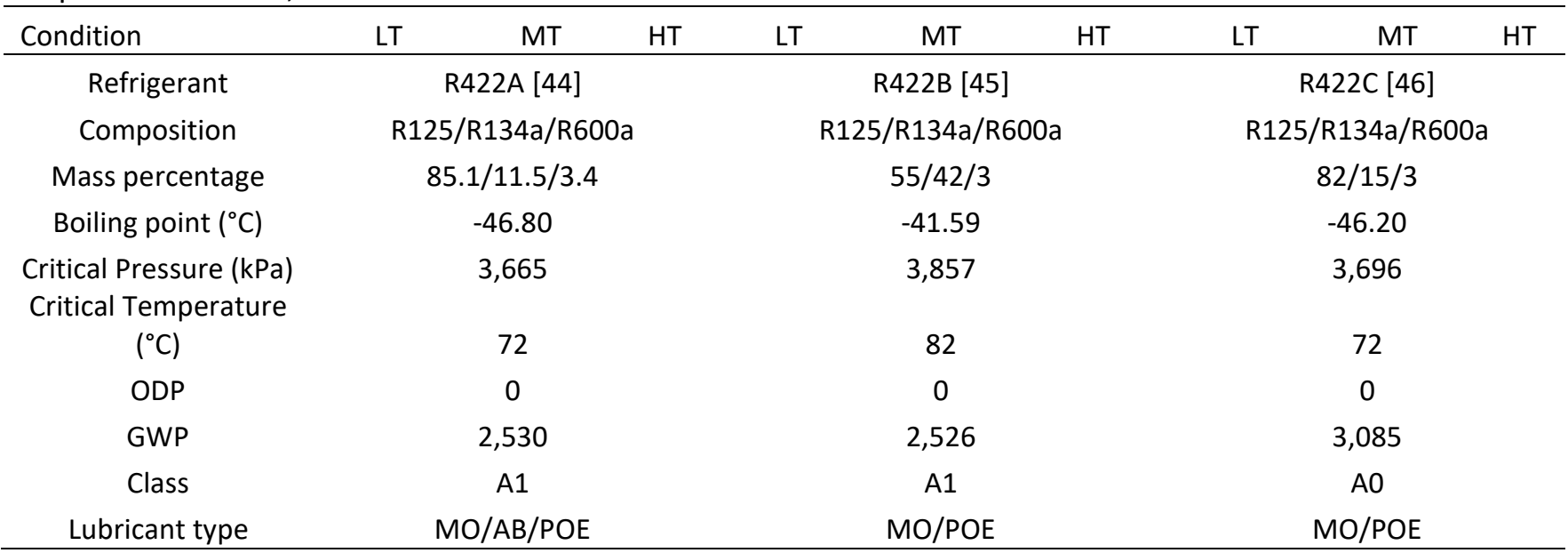

Table 3

Properties of R422D, R424A and R437A

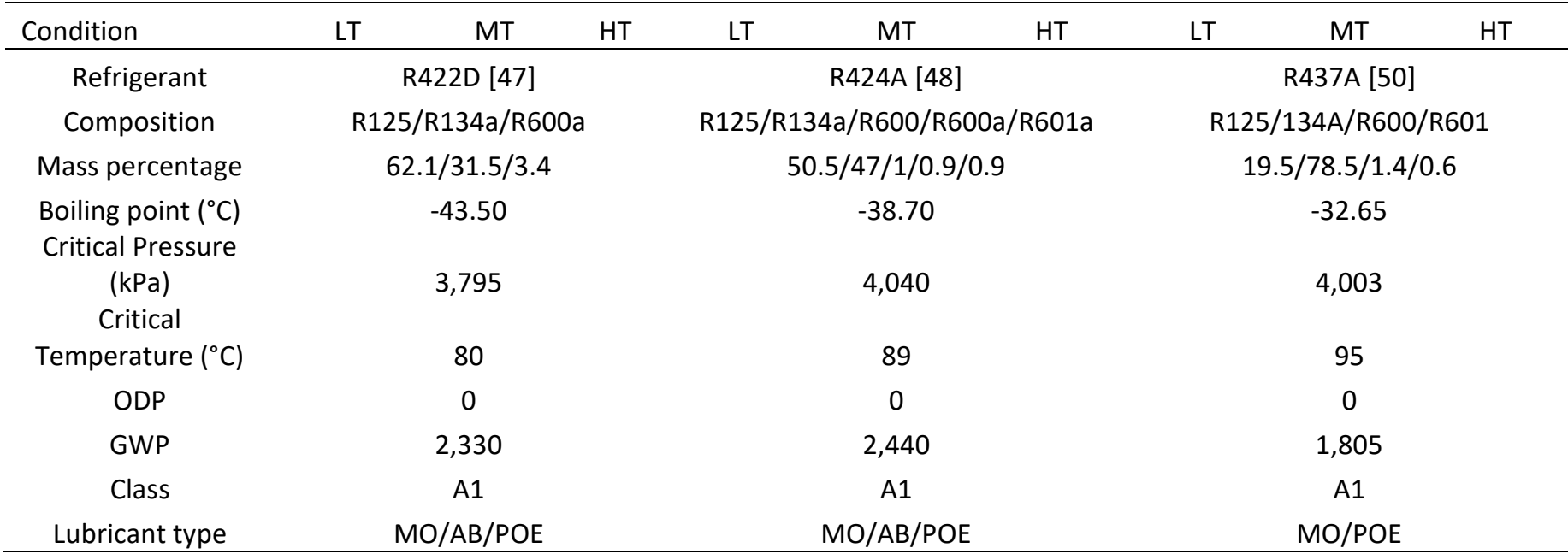

Table 4

Properties of R438A and R453A

\begin{tabular}{|c|c|c|c|c|}
\hline Condition & MT & $\mathrm{HT}$ & MT & HT \\
\hline Refrigerant & \multicolumn{2}{|c|}{ R438A [51] } & \multicolumn{2}{|c|}{ R R453A [52] } \\
\hline Composition & \multicolumn{2}{|c|}{ R125/134A/R32/R600/R601a } & \multicolumn{2}{|c|}{ R125/R32/R134A/R227ea/R600/R601A } \\
\hline Mass percentage & \multicolumn{2}{|c|}{$45 / 44.2 / 8.5 / 1.7 / 0.6$} & \multicolumn{2}{|c|}{ 20/20/53.8/5/0.6/0.6 } \\
\hline Boiling point $\left({ }^{\circ} \mathrm{C}\right)$ & \multicolumn{2}{|c|}{-42.61} & \multicolumn{2}{|c|}{-42.20} \\
\hline \multicolumn{5}{|l|}{ Critical Pressure } \\
\hline$(\mathrm{kPa})$ & \multicolumn{2}{|c|}{4,179} & \multicolumn{2}{|l|}{4,530} \\
\hline \multicolumn{5}{|l|}{ Critical Temperature } \\
\hline$\left({ }^{\circ} \mathrm{C}\right)$ & \multicolumn{2}{|l|}{84} & \multicolumn{2}{|l|}{88} \\
\hline ODP & \multicolumn{2}{|l|}{0} & \multicolumn{2}{|l|}{0} \\
\hline GWP & \multicolumn{2}{|c|}{2,265} & \multicolumn{2}{|l|}{1,765} \\
\hline Class & \multicolumn{2}{|l|}{ AO } & \multicolumn{2}{|l|}{ A1 } \\
\hline Lubricant type & \multicolumn{2}{|c|}{ MO/POE } & \multicolumn{2}{|c|}{ MO/POE } \\
\hline
\end{tabular}




\section{Table 5}

Properties of R134A, R450A, R456A, R513A and R515A

\begin{tabular}{|c|c|c|c|c|c|}
\hline Refrigerant & R134A [53] & R450A [54] & R456A [40] & R513A [55] & R515A [40] \\
\hline Composition & R134A & $\begin{array}{c}\text { R134A/ } \\
\text { R12354ze(E) }\end{array}$ & $\begin{array}{c}\text { R134a/R32/R1234ze } \\
\text { (E) }\end{array}$ & $\begin{array}{l}\text { R134A/ } \\
\text { R1234yf }\end{array}$ & $\begin{array}{l}\text { R227ea/ } \\
\text { R1234ze }\end{array}$ \\
\hline Mass percentage & 100 & $42 / 58$ & $45 / 6 / 49$ & $44 / 56$ & $12 / 88$ \\
\hline Boiling point $\left({ }^{\circ} \mathrm{C}\right)$ & -26.07 & -23.5 & -30.75 & -28.3 & -18.75 \\
\hline Critical pressure $(\mathrm{kPa})$ & 4060 & 3814 & 4175 & 3700 & 3555 \\
\hline Critical temperature $\left({ }^{\circ} \mathrm{C}\right)$ & 101.06 & 105.87 & 102.65 & 97.7 & 108.65 \\
\hline ODP & 0 & 0 & 0 & 0 & 0 \\
\hline GWP & 1430 & 547 & 687 & 570 & 387 \\
\hline Class & A1 & A1 & A1 & A1 & A1 \\
\hline Lubricant type & POE & POE & POE & POE & POE \\
\hline
\end{tabular}

\section{Table 6}

Properties of R404A, R407A, and R407F

\begin{tabular}{|c|c|c|c|c|c|c|c|c|c|}
\hline Condition & LT & MT & HT & LT & MT & HT & LT & MT & HT \\
\hline Refrigerant & \multicolumn{3}{|c|}{ R404A [56] } & \multicolumn{3}{|c|}{ R407A [57] } & \multicolumn{3}{|c|}{ R407F [58] } \\
\hline Composition & \multicolumn{3}{|c|}{ R125/R143/R134A } & \multicolumn{3}{|c|}{ R125/R32/R134A } & \multicolumn{3}{|c|}{ R125/R32/R134A } \\
\hline Mass percentage & \multicolumn{3}{|c|}{$44 / 52 / 4$} & \multicolumn{3}{|c|}{$40 / 20 / 40$} & \multicolumn{3}{|c|}{$30 / 30 / 40$} \\
\hline Boiling point $\left({ }^{\circ} \mathrm{C}\right)$ at $1 \mathrm{kPa}$ & \multicolumn{3}{|c|}{-46.6} & \multicolumn{3}{|c|}{-45.28} & \multicolumn{3}{|c|}{-46.33} \\
\hline Critical pressure $(\mathrm{kPa})$ & \multicolumn{3}{|c|}{3728} & \multicolumn{3}{|c|}{4494} & \multicolumn{3}{|c|}{4754} \\
\hline Critical temperature $\left({ }^{\circ} \mathrm{C}\right)$ & \multicolumn{3}{|c|}{72.1} & \multicolumn{3}{|c|}{82} & \multicolumn{3}{|c|}{82.6} \\
\hline ODP & \multicolumn{3}{|c|}{0} & \multicolumn{3}{|c|}{0} & \multicolumn{3}{|c|}{0} \\
\hline GWP & \multicolumn{3}{|c|}{3943} & \multicolumn{3}{|c|}{2107} & \multicolumn{3}{|c|}{1825} \\
\hline Class & \multicolumn{3}{|c|}{$\mathrm{A} 1$} & \multicolumn{3}{|c|}{ A1 } & \multicolumn{3}{|c|}{ A1 } \\
\hline Lubricant type & \multicolumn{3}{|c|}{ POE } & \multicolumn{3}{|c|}{ POE } & \multicolumn{3}{|c|}{ POE } \\
\hline Qevap (kJ/kg) & \multicolumn{3}{|c|}{83.66} & \multicolumn{3}{|c|}{139.02} & \multicolumn{3}{|c|}{$\mathrm{N} / \mathrm{A}$} \\
\hline Qcond $(\mathrm{kJ} / \mathrm{kg})$ & \multicolumn{3}{|c|}{159.8} & \multicolumn{3}{|c|}{198.57} & & $\mathrm{~N} / \mathrm{A}$ & \\
\hline Work $(\mathrm{kJ} / \mathrm{kg})$ & & 6.14 & & & 59.55 & & & $\mathrm{~N} / \mathrm{A}$ & \\
\hline COPc & & 099 & & & 2.335 & & & N/A & \\
\hline Evaporator pressure $(\mathrm{kPa})$ & & 33.30 & & & 477.3 & & & N/A & \\
\hline Condenser pressure (kPa) & & $\begin{array}{r}97.50 \\
\end{array}$ & & & 2284.10 & & & $\mathrm{~N} / \mathrm{A}$ & \\
\hline Evaporator temp glide $\left({ }^{\circ} \mathrm{C}\right)$ & -0.4 & 0.5 & $\mathrm{~N} / \mathrm{A}$ & 119.21 & 126.89 & 114.83 & 192.46 & 184.93 & 170.29 \\
\hline Condenser temp glide $\left({ }^{\circ} \mathrm{C}\right)$ & 0.3 & 0.3 & N/A & 216.04 & 189.24 & 166.05 & 328.41 & 266.99 & 237.2 \\
\hline
\end{tabular}

Table 7

Properties of R407H, R410A, and R422A

\begin{tabular}{|c|c|c|c|c|c|c|c|c|c|}
\hline Condition & LT & MT & HT & LT & $\mathrm{MT}$ & HT & LT & MT & $\mathrm{HT}$ \\
\hline \multirow[t]{2}{*}{ Refrigerant } & \multirow{2}{*}{\multicolumn{3}{|c|}{ R407H [59] }} & \multirow{2}{*}{\multicolumn{2}{|c|}{ R410A [60] }} & \multicolumn{4}{|c|}{ R442A [52] } \\
\hline & & & & & & \multicolumn{4}{|c|}{ R125/R32/R1234A } \\
\hline Composition & \multicolumn{3}{|c|}{ R125/R32/R134A } & \multicolumn{3}{|c|}{ R125/R32 } & \multicolumn{3}{|c|}{ /R227ea/R152A } \\
\hline Mass percentage & \multicolumn{3}{|c|}{$15 / 32.5 / 52.5$} & \multicolumn{3}{|c|}{$50 / 50$} & \multicolumn{3}{|c|}{$31 / 31 / 30 / 5 / 3$} \\
\hline Boiling point $\left({ }^{\circ} \mathrm{C}\right)$ & \multirow{2}{*}{\multicolumn{3}{|c|}{-44.6}} & \multirow{2}{*}{\multicolumn{2}{|c|}{-51.6}} & & \multicolumn{3}{|c|}{-46.5} \\
\hline Critical pressure & & & & & & & & & \\
\hline$(\mathrm{kPa})$ & \multicolumn{3}{|c|}{4856} & \multicolumn{3}{|c|}{4811} & \multicolumn{3}{|c|}{4760} \\
\hline
\end{tabular}


Critical temperature

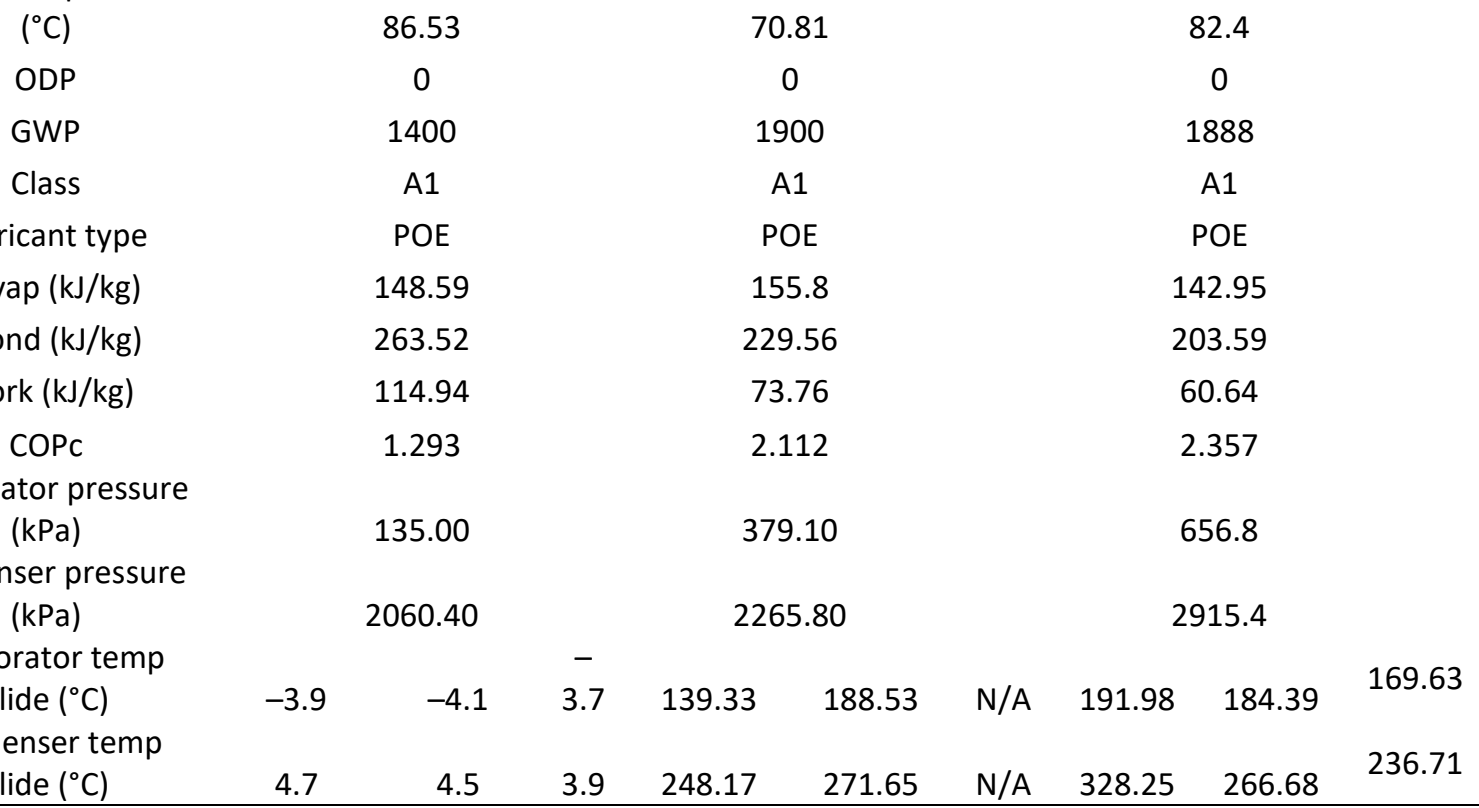

\section{Table 8}

Properties of R448A, R449A, and R452A

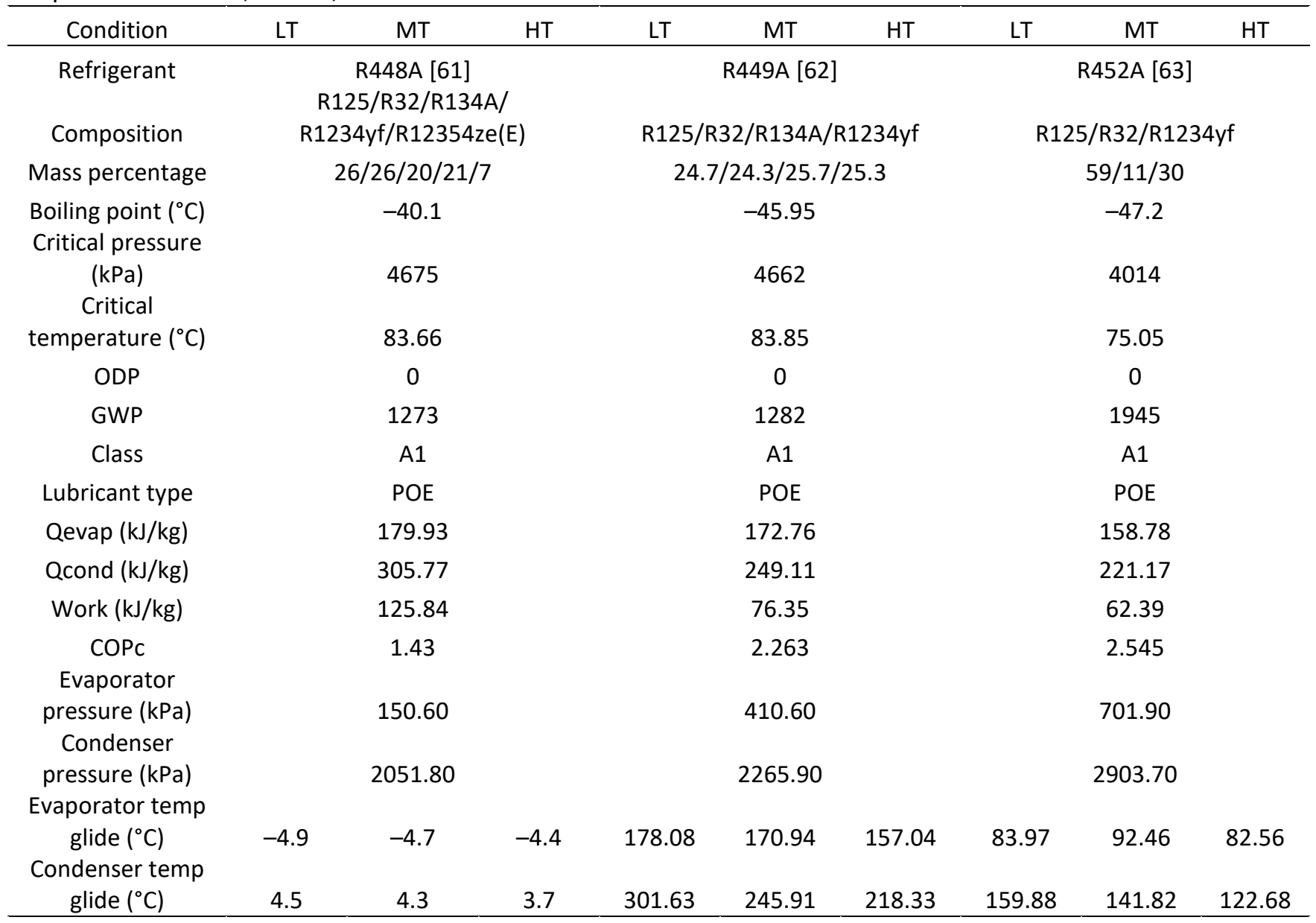




\section{Table 9}

Properties of R453A and R463A

\begin{tabular}{|c|c|c|c|c|c|c|}
\hline Condition & LT & MT & HT & LT & MT & HT \\
\hline Refrigerant & \multicolumn{3}{|c|}{ R453A [64], } & \multicolumn{3}{|c|}{ R463A [65] } \\
\hline & \multicolumn{3}{|c|}{ R125/R32/R134A/R227ea/ } & & & \\
\hline Composition & & $0 /$ R60 & & \multicolumn{3}{|c|}{ R125/R32/R134A/R1234yf/R744 } \\
\hline Mass percentage & \multicolumn{3}{|c|}{ 20/20/53.8/5/0.6/0.6 } & \multicolumn{3}{|c|}{$30 / 36 / 14 / 14 / 6$} \\
\hline Boiling point $\left({ }^{\circ} \mathrm{C}\right)$ & \multicolumn{3}{|c|}{-42.2} & \multicolumn{3}{|c|}{-60.13} \\
\hline Critical pressure ( $\mathrm{kPa})$ & \multicolumn{3}{|c|}{4530} & \multicolumn{3}{|c|}{5283} \\
\hline Critical temperature $\left({ }^{\circ} \mathrm{C}\right)$ & \multicolumn{3}{|c|}{87.9} & \multicolumn{3}{|c|}{73.15} \\
\hline ODP & \multicolumn{3}{|c|}{0} & \multicolumn{3}{|c|}{0} \\
\hline GWP & \multicolumn{3}{|c|}{1765} & \multicolumn{3}{|c|}{1377} \\
\hline Class & \multicolumn{3}{|c|}{ A1 } & \multicolumn{3}{|c|}{ A1 } \\
\hline Lubricant type & \multicolumn{3}{|c|}{ POE } & \multicolumn{3}{|c|}{ POE } \\
\hline Qevap (kJ/kg) & \multicolumn{3}{|c|}{184.91} & \multicolumn{3}{|c|}{178.36} \\
\hline Qcond $(\mathrm{kJ} / \mathrm{kg})$ & \multicolumn{3}{|c|}{312} & \multicolumn{3}{|c|}{255.92} \\
\hline Work $(\mathrm{kJ} / \mathrm{kg})$ & \multicolumn{3}{|c|}{127.56} & \multicolumn{3}{|c|}{77.56} \\
\hline COPc & \multicolumn{3}{|c|}{1.45} & \multicolumn{3}{|c|}{2.3} \\
\hline Evaporator pressure $(\mathrm{kPa})$ & \multicolumn{3}{|c|}{121.00} & \multicolumn{3}{|c|}{342.10} \\
\hline Condenser pressure (kPa) & \multicolumn{3}{|c|}{1808.70} & \multicolumn{3}{|c|}{2002.50} \\
\hline Evaporator temp glide $\left({ }^{\circ} \mathrm{C}\right)$ & -5.2 & -5.1 & 165.49 & 194.65 & 186.07 & 168.25 \\
\hline Condenser temp glide $\left({ }^{\circ} \mathrm{C}\right)$ & 5.0 & 4.8 & 228.96 & 340.43 & 273.5 & 239.3 \\
\hline
\end{tabular}

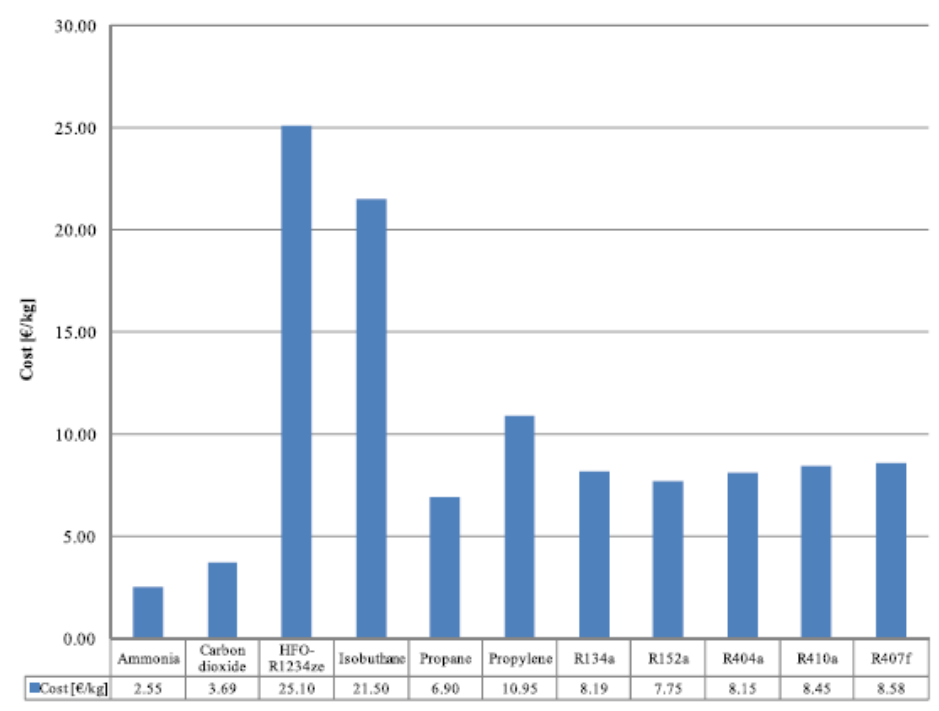

Fig. 6. Cost of refrigerant [43]

For the cost of refrigerant as shown in Figure 6 [43], the refrigerant should be mixed with HFOs. It also highest refrigerant cost but does not include HCs refrigerant cost compare with HFOs refrigerant cost, which is being presented as a refrigerant for comparative purposes in this research as it is composed of HCs. The properties of hydrocarbon refrigerant that class A3 high flammability as shown in Figure 7 below but zero ODP and GWP nearly zero, shown in Table 10- 
11 for R170 [66], R290 [67], R600 [68], R600a [69] and Table 2 for R601 [70], R601a [71], R1150 [72], R1270 [73]. The highest boiling of R170 and R1270 were found to be $-88.70^{\circ} \mathrm{C}$ and $-103.8^{\circ} \mathrm{C}$ respectively but the critical temperature was found to be $32.17^{\circ} \mathrm{C}$ and $9.5^{\circ} \mathrm{C}$. This means those are refrigerant that cannot operated in refrigerant in accordance with the CAN/ANSI/AHRI540 Air-Conditioning, Heating, and Refrigeration Institute (AHRI) standards that standard for this research [74-76]. The R290 and R1270 were found to be nearly boiling point with R22 at $-42.1 .{ }^{\circ} \mathrm{C}$ and $-47.7^{\circ} \mathrm{C}$ respectively but operate at high condenser pressure that will affect to refrigerant work and cooling coefficient of performance.

\section{Table 10}

Properties of R170, R290, R600, R600a

\begin{tabular}{|c|c|c|c|c|c|c|c|c|c|c|c|c|}
\hline Condition & LT & MT & $\mathrm{HT}$ & LT & MT & HT & LT & MT & HT & LT & MT & HT \\
\hline Refrigerant & & R170 [66 & & & R290 [67] & & & R600 [68] & & & R600a [69] & \\
\hline Formula & & $\mathrm{C} 2 \mathrm{H} 6$ & & & $\mathrm{C} 3 \mathrm{H} 8$ & & & $\mathrm{C} 4 \mathrm{H} 10$ & & & $\mathrm{C} 4 \mathrm{H} 10$ & \\
\hline $\begin{array}{l}\text { Chemical } \\
\text { name } \\
\text { Boiling point }\end{array}$ & & Ethane & & & Propane & & & Butane & & & Isobutane & \\
\hline $\begin{array}{l}\left({ }^{\circ} \mathrm{C}\right) \\
\text { Critical }\end{array}$ & & -88.7 & & & -42.1 & & & -0.5 & & & -11.73 & \\
\hline $\begin{array}{l}\text { Pressure } \\
(\mathrm{kPa}) \\
\text { Critical }\end{array}$ & & 4872 & & & 4251 & & & 3796 & & & 3629 & \\
\hline Temperature & & & & & & & & & & & & \\
\hline$\left({ }^{\circ} \mathrm{C}\right)$ & & 32.17 & & & 96.74 & & & 151.98 & & & 134.66 & \\
\hline ODP & & 0 & & & 0 & & & 0 & & & 0 & \\
\hline GWP & & 3 & & & 3 & & & 3 & & & 3 & \\
\hline $\begin{array}{c}\text { Class } \\
\text { Lubricant }\end{array}$ & & A3 & & & A3 & & & A3 & & & A3 & \\
\hline type & & $\mathrm{MO} / \mathrm{POE}$ & & & $\mathrm{MO} / \mathrm{POE}$ & & & MO/POE & & & MO/POE & \\
\hline $\begin{array}{l}\text { (kJ/kg) } \\
\text { Qcond }\end{array}$ & N/A & N/A & N/A & 388.96 & 240.37 & 223.89 & 235.72 & 261.99 & 255.88 & 207.03 & 231.52 & 223.97 \\
\hline (kJ/kg) & N/A & $\mathrm{N} / \mathrm{A}$ & N/A & 221.85 & 349.48 & 314.59 & 400.21 & 371.49 & 348.29 & 358.39 & 332.01 & 308.46 \\
\hline Work (kJ/kg) & N/A & $\mathrm{N} / \mathrm{A}$ & N/A & 221.85 & 109.11 & 90.70 & 164.50 & 109.51 & 92.41 & 151.35 & 100.49 & 84.49 \\
\hline $\begin{array}{c}\text { COPc } \\
\text { Evaporator } \\
\text { Pressure }\end{array}$ & N/A & $\mathrm{N} / \mathrm{A}$ & N/A & 1.33 & 2.20 & 2.47 & 1.43 & 2.39 & 2.77 & 1.37 & 2.30 & 2.65 \\
\hline $\begin{array}{c}(\mathrm{kPa}) \\
\text { Condenser } \\
\text { Pressure }\end{array}$ & N/A & $\mathrm{N} / \mathrm{A}$ & N/A & 157.70 & 385.90 & 623.90 & 26.20 & 80.20 & 145.60 & 43.30 & 123.50 & 216.00 \\
\hline$(\mathrm{kPa})$ & N/A & $\mathrm{N} / \mathrm{A}$ & N/A & 1653.10 & 1803.10 & 2269.40 & 484.30 & 535.40 & 705.00 & 670.60 & 736.80 & 955.00 \\
\hline
\end{tabular}




\section{Table 11}

Properties of R601, R601a, R1150, R1270

\begin{tabular}{|c|c|c|c|c|c|c|c|c|c|c|c|c|}
\hline Condition & LT & MT & $\mathrm{HT}$ & $\mathrm{LT}$ & MT & $\mathrm{HT}$ & $\mathrm{LT}$ & MT & $\mathrm{HT}$ & LT & MT & $\mathrm{HT}$ \\
\hline Refrigerant & \multicolumn{3}{|c|}{ R601 [70] } & \multicolumn{3}{|c|}{ R601a [71] } & \multicolumn{3}{|c|}{ R1150 [72] } & \multicolumn{3}{|c|}{ R1270 [73] } \\
\hline Formula & \multicolumn{3}{|c|}{$\mathrm{C} 5 \mathrm{H} 12$} & \multicolumn{3}{|c|}{$\mathrm{C} 5 \mathrm{H} 12$} & \multicolumn{3}{|c|}{$\mathrm{C} 2 \mathrm{H} 4$} & \multicolumn{3}{|c|}{$\mathrm{C} 3 \mathrm{H} 6$} \\
\hline Chemical name & \multicolumn{3}{|c|}{ Pentane } & \multicolumn{3}{|c|}{ Isopentane } & \multicolumn{3}{|c|}{ Ethylene } & \multicolumn{3}{|c|}{ Propylene } \\
\hline Boiling point $\left({ }^{\circ} \mathrm{C}\right)$ & \multicolumn{3}{|c|}{36.1} & \multicolumn{3}{|c|}{27.7} & \multicolumn{3}{|c|}{-103.8} & \multicolumn{3}{|c|}{-47.7} \\
\hline $\begin{array}{c}\text { Critical Pressure } \\
(\mathrm{kPa}) \\
\text { Critical }\end{array}$ & \multicolumn{3}{|c|}{3370} & \multicolumn{3}{|c|}{3378} & & \multicolumn{2}{|c|}{5042} & & 4660 & \\
\hline Temperature $\left({ }^{\circ} \mathrm{C}\right)$ & \multicolumn{3}{|c|}{196.55} & \multicolumn{3}{|c|}{187.2} & \multicolumn{3}{|c|}{9.5} & \multicolumn{3}{|c|}{92.4} \\
\hline ODP & \multicolumn{3}{|c|}{0} & \multicolumn{3}{|c|}{0} & \multicolumn{3}{|c|}{0} & \multicolumn{3}{|c|}{0} \\
\hline GWP & \multicolumn{3}{|c|}{4} & \multicolumn{3}{|c|}{4} & \multicolumn{3}{|c|}{3} & \multicolumn{3}{|c|}{2} \\
\hline Class & \multicolumn{3}{|c|}{ A3 } & & $\mathrm{A} 3$ & & & A3 & & & A3 & \\
\hline Lubricant type & & $\mathrm{MO} / \mathrm{POE}$ & & & MO/POE & & & 10/PC & & & MO/POE & \\
\hline & 239.3 & 267.2 & 264.1 & 221.1 & 248.0 & 244.6 & $\mathrm{~N} /$ & $\mathrm{N} /$ & $\mathrm{N} /$ & & & \\
\hline Qevap (kJ/kg) & $\begin{array}{c}0 \\
402.1\end{array}$ & $\begin{array}{c}2 \\
376.3\end{array}$ & $\begin{array}{c}3 \\
356.7\end{array}$ & $\begin{array}{c}8 \\
374.8\end{array}$ & $\begin{array}{c}5 \\
350.9\end{array}$ & $\begin{array}{c}5 \\
331.9\end{array}$ & $\begin{array}{c}\mathrm{A} \\
\mathrm{N} /\end{array}$ & $\begin{array}{c}\mathrm{A} \\
\mathrm{N} /\end{array}$ & $\begin{array}{c}\mathrm{A} \\
\mathrm{N} /\end{array}$ & 232.45 & 247.13 & 228.13 \\
\hline Qcond (kJ/kg) & $\begin{array}{c}6 \\
162.8\end{array}$ & $\begin{array}{c}1 \\
109.0\end{array}$ & 2 & $\begin{array}{c}1 \\
153.6\end{array}$ & $\begin{array}{c}9 \\
102.9\end{array}$ & 5 & $\begin{array}{c}\mathrm{A} \\
\mathrm{N} /\end{array}$ & $\begin{array}{c}\mathrm{A} \\
\mathrm{N} /\end{array}$ & $\begin{array}{c}\mathrm{A} \\
\mathrm{N} /\end{array}$ & 404.89 & 358.77 & 320.62 \\
\hline Work (kJ/kg) & 6 & 9 & 92.59 & 3 & 4 & 87.30 & $\begin{array}{c}\mathrm{A} \\
\mathrm{N} /\end{array}$ & $\begin{array}{c}\mathrm{A} \\
\mathrm{N} /\end{array}$ & $\begin{array}{c}\mathrm{A} \\
\mathrm{N} /\end{array}$ & 172.44 & 111.64 & 92.48 \\
\hline $\begin{array}{c}\text { COPc } \\
\text { Evaporator }\end{array}$ & 1.47 & 2.45 & 2.85 & 1.44 & 2.41 & 2.80 & $\begin{array}{c}\mathrm{A} \\
\mathrm{N} /\end{array}$ & $\begin{array}{c}\mathrm{A} \\
\mathrm{N} /\end{array}$ & $\begin{array}{c}\mathrm{A} \\
\mathrm{N} /\end{array}$ & 1.35 & 2.21 & 2.47 \\
\hline Pressure (kPa) & 4.70 & 18.10 & 37.00 & 7.20 & 25.90 & 51.20 & $A$ & $A$ & $A$ & 199.70 & 478.10 & 764.40 \\
\hline Condenser & 155.8 & 175.1 & 242.2 & 201.7 & 225.4 & 307.0 & $\mathrm{~N} /$ & N/ & $\mathrm{N} /$ & 1964.9 & 2143.8 & 2686.7 \\
\hline Pressure $(\mathrm{kPa})$ & 0 & 0 & 0 & 0 & 0 & 0 & $A$ & A & $A$ & 0 & 0 & 0 \\
\hline
\end{tabular}

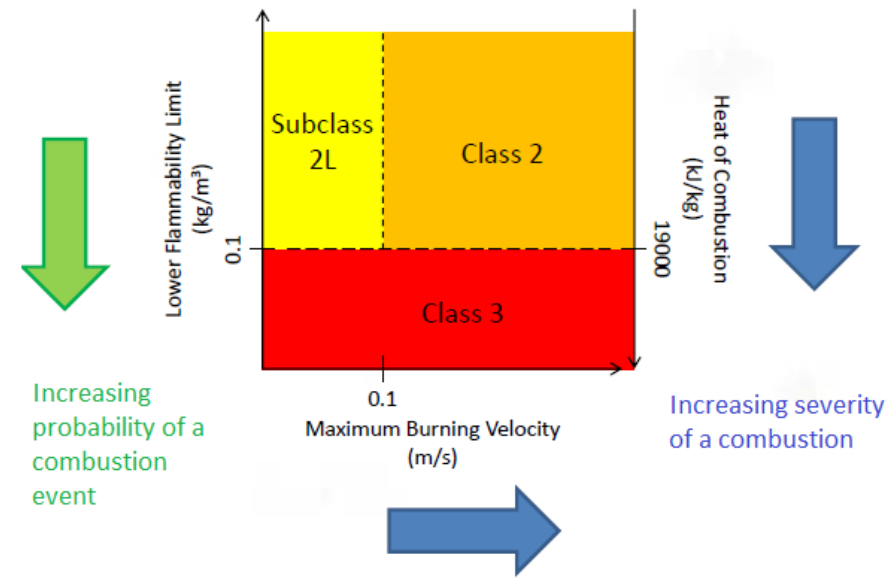

Fig. 7. Refrigerant classification [2]

\section{Methodology}

The medium back pressure refrigeration testing standard that follow CAN/ANSI/AHRI540 standard air-conditioning, heating, and refrigeration institute (AHRI) that showed in Table 12 as below. [74-76]. And the datamining tool for select refrigerants mass percentage that used rapid minor software that first of report of the software poll that used for data mining tool in Figure 8 as below. [77]. The Properties of refrigerants and refrigeration simulation system that used REFPROP 
and CYCLE_D-HX software from national institute of standards and technology [78-80] in Figure 9 as below.

\section{Table 12}

Standard testing for refrigeration systems [74-76]

\begin{tabular}{lccccc}
\hline \multirow{2}{*}{ Temperature Point } & \multicolumn{3}{c}{ Air Conditioning and Heat Pump } & \multicolumn{3}{c}{ Refrigeration } \\
\cline { 2 - 6 } & Heating & Cooling & Low & Medium & High \\
\hline Suction dew point $\left({ }^{\circ} \mathrm{C}\right)$ & -15.0 & 10.0 & -31.5 & -6.5 & 7.0 \\
Discharge dew point $\left({ }^{\circ} \mathrm{C}\right)$ & 35.0 & 46.0 & 40.5 & 43.5 & 54.5 \\
Suction return gas temperature $\left({ }^{\circ} \mathrm{C}\right)$ & -4.0 & 21.0 & 4.5 & 18.5 & 18.5 \\
Superheat $(\mathrm{K})$ & 11.0 & 11.0 & 11.0 & 11.0 & 11.0 \\
Subcooling $(\mathrm{K})$ & 0.0 & 0.0 & 0.0 & 0.0 & 0.0 \\
\hline
\end{tabular}

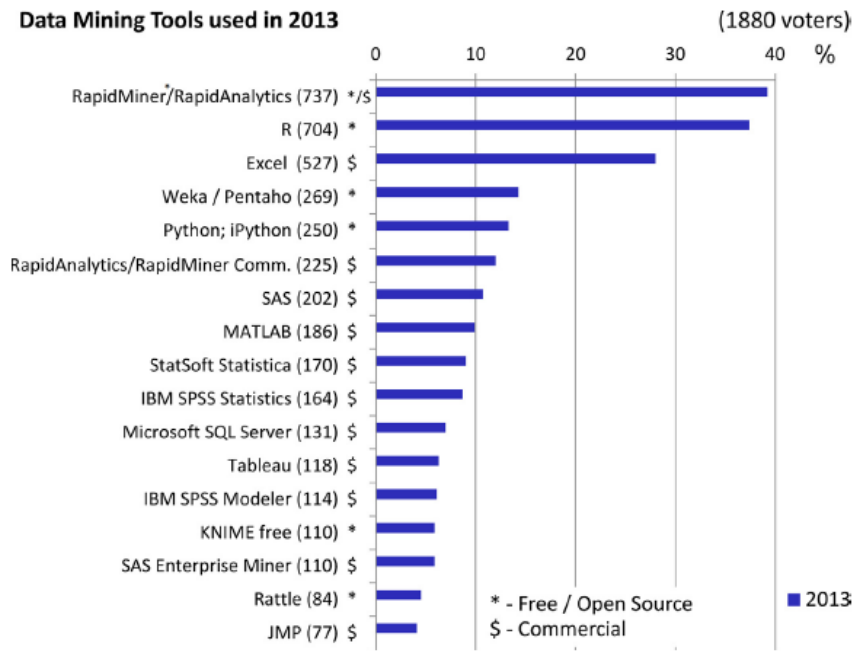

Fig 3. Report of the KDnuggets Annual Software Poll: Using data mining tools in 2013xps13\#(1880 voters, KDnuggets Annual Software Poll, 2013).

Fig. 8. Report of the software poll that used for data mining tool [77]

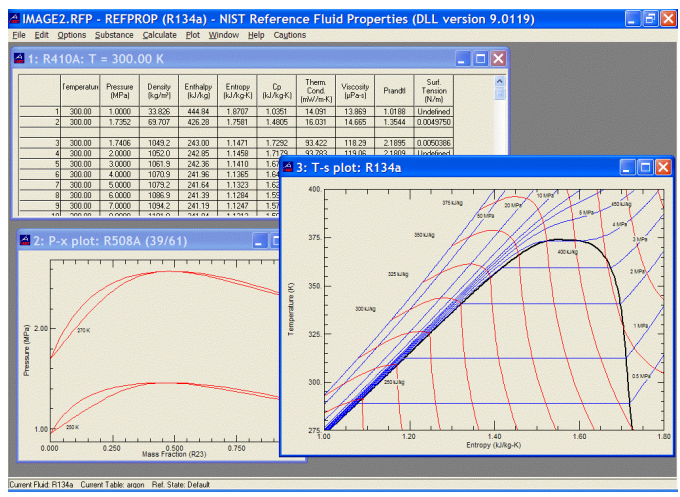

Fig. 9. The REFPROP software for refrigerant properties [78-80] 


\section{Results and Discussion}

The refrigerants mixed by R134, R32, R125 and R1270 that can happen 4,539 type in Table 13 as below has showed the refrigerants mixed by R134, R32, R125 and R1270 by fixed R1270 in 1\% for refrigerant no frame propagation class $A 1$ and varies mass percentage of R134, R32 and R125 and the refrigerants mixed class A2 that mass percentage of R32 higher than R125 The GWP calculated by mass percentage of refrigerants and boiling point simulate by REFPROP software at $0.1 \mathrm{Mpa}$ and pressure at evaporator temperature $-6.5^{\circ} \mathrm{C}$ and pressure at condenser temperature $43.5^{\circ} \mathrm{C}$ simulate by and CYCLE_D-HX software from national institute of standards and technology [19] The refrigerant mixed were developed to low GWP, zero ODP, high capacity, low pressure, no toxify [6] that can select the refrigerant requirement from refrigerant data 4,539 type by rapid minor software that first of report of the software poll that used for data mining tool. The refrigerant requirement was class A1, boiling point lower than boiling point R404 at $-46.5^{\circ} \mathrm{C}$ and GWP lower than 2000 . The datamining functional by decision tree in rapid minor software showed the 4 refrigerant result that follow refrigerant requirement as Figure 10, 11 and Table 14 below.

Table 13

The refrigerants mixed by R134, R32, R125 and R1270

\begin{tabular}{|c|c|c|c|c|c|c|c|c|}
\hline Refrigerant & $\mathrm{R} 134 \mathrm{~A}$ & R32 & R125 & $\mathrm{R} 1270$ & Summary & GWP & Boiling Point & Class A \\
\hline R-No.1 & 1 & 1 & 97 & 1 & 100 & 3,366 & -47.91 & 1 \\
\hline R-No.2 & 1 & 2 & 96 & 1 & 100 & 3,339 & -47.95 & 1 \\
\hline R-No.3 & 1 & 3 & 95 & 1 & 100 & 3,311 & -47.98 & 1 \\
\hline R-No.4 & 1 & 4 & 94 & 1 & 100 & 3,283 & -48.02 & 1 \\
\hline$\bullet$ & $\bullet$ & $\bullet$ & $\bullet$ & $\bullet$ & $\bullet$ & $\bullet$ & $\bullet$ & $\bullet$ \\
\hline R-No.94 & 1 & 94 & 4 & 1 & 100 & 786 & -51.26 & 2 \\
\hline R-No.95 & 1 & 95 & 3 & 1 & 100 & 758 & -51.30 & 2 \\
\hline R-No.96 & 1 & 96 & 2 & 1 & 100 & 730 & -51.33 & 2 \\
\hline R-No.97 & 2 & 3 & 94 & 1 & 100 & 3,289 & -47.76 & 1 \\
\hline R-No.98 & 2 & 4 & 93 & 1 & 100 & 3,262 & -47.80 & 1 \\
\hline R-No.99 & 2 & 5 & 92 & 1 & 100 & 3,234 & -47.84 & 1 \\
\hline R-No.100 & 2 & 6 & 91 & 1 & 100 & 3,206 & -47.87 & 1 \\
\hline$\bullet$ & $\bullet$ & $\bullet$ & $\bullet$ & $\bullet$ & $\bullet$ & $\bullet$ & $\bullet$ & $\bullet$ \\
\hline R-No.187 & 2 & 93 & 4 & 1 & 100 & 792 & -51.00 & 2 \\
\hline R-No.188 & 2 & 94 & 3 & 1 & 100 & 764 & -51.04 & 2 \\
\hline R-No.189 & 2 & 95 & 2 & 1 & 100 & 736 & -51.08 & 2 \\
\hline R-No.190 & 3 & 3 & 93 & 1 & 100 & 3,268 & -47.54 & 1 \\
\hline R-No.191 & 3 & 4 & 92 & 1 & 100 & 3,240 & -47.58 & 1 \\
\hline R-No.192 & 3 & 5 & 91 & 1 & 100 & 3,212 & -47.62 & 1 \\
\hline$\bullet$ & $\bullet$ & $\bullet$ & $\bullet$ & $\bullet$ & $\bullet$ & $\bullet$ & $\bullet$ & $\bullet$ \\
\hline R-No.4534 & 92 & 3 & 4 & 1 & 100 & 1,354 & -27.96 & 1 \\
\hline R-No.4535 & 92 & 4 & 3 & 1 & 100 & 1,327 & -28.00 & 2 \\
\hline R-No.4536 & 92 & 5 & 2 & 1 & 100 & 1,299 & -28.04 & 2 \\
\hline R-No.4537 & 93 & 3 & 3 & 1 & 100 & 1,333 & -27.74 & 1 \\
\hline R-No.4538 & 94 & 3 & 2 & 1 & 100 & 1,311 & -27.52 & 2 \\
\hline R-No.4539 & 95 & 3 & 1 & 1 & 100 & 1,290 & -27.30 & 2 \\
\hline
\end{tabular}




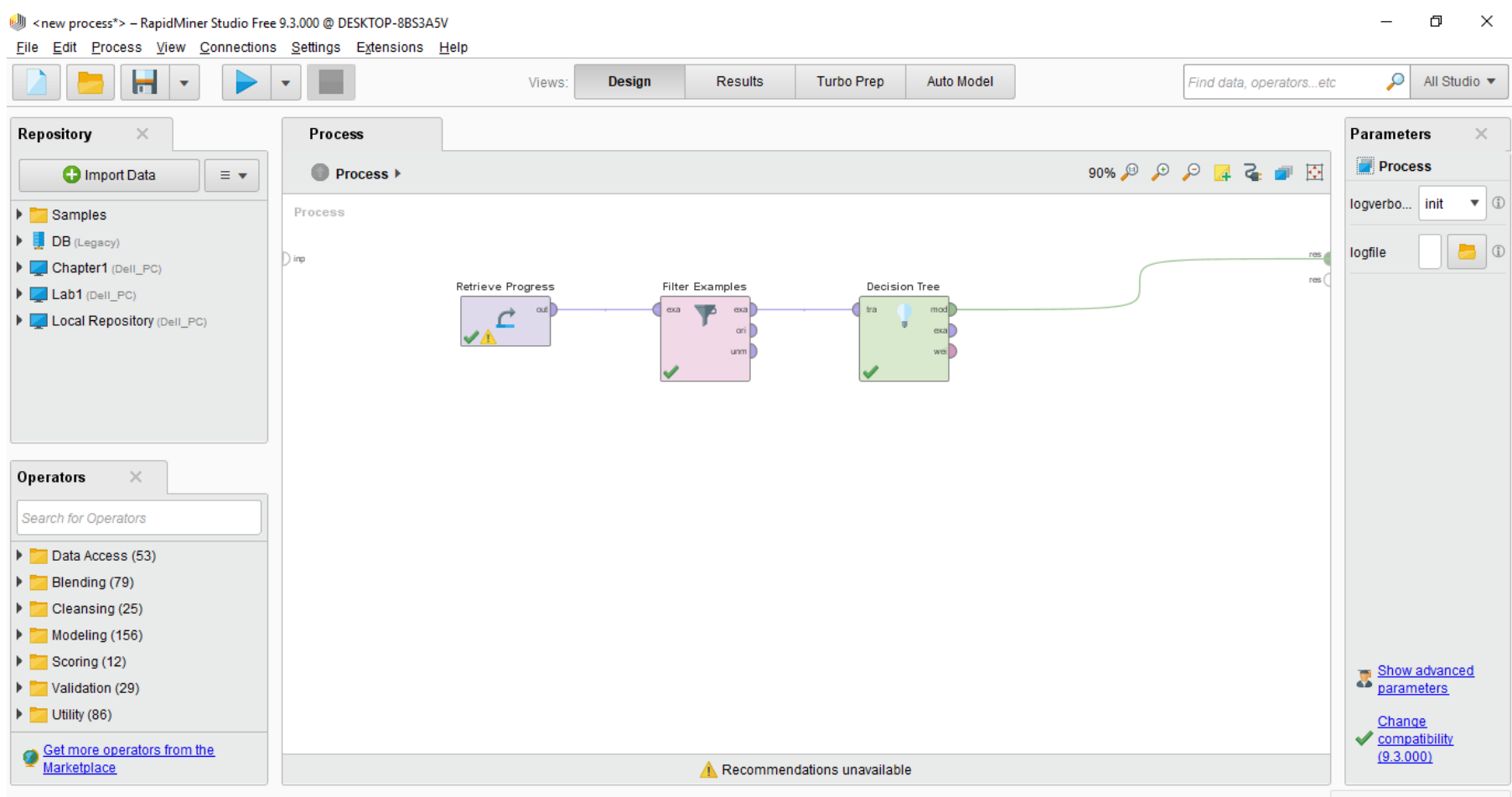

Fig.10. The datamining functional by decision tree in rapid minor software

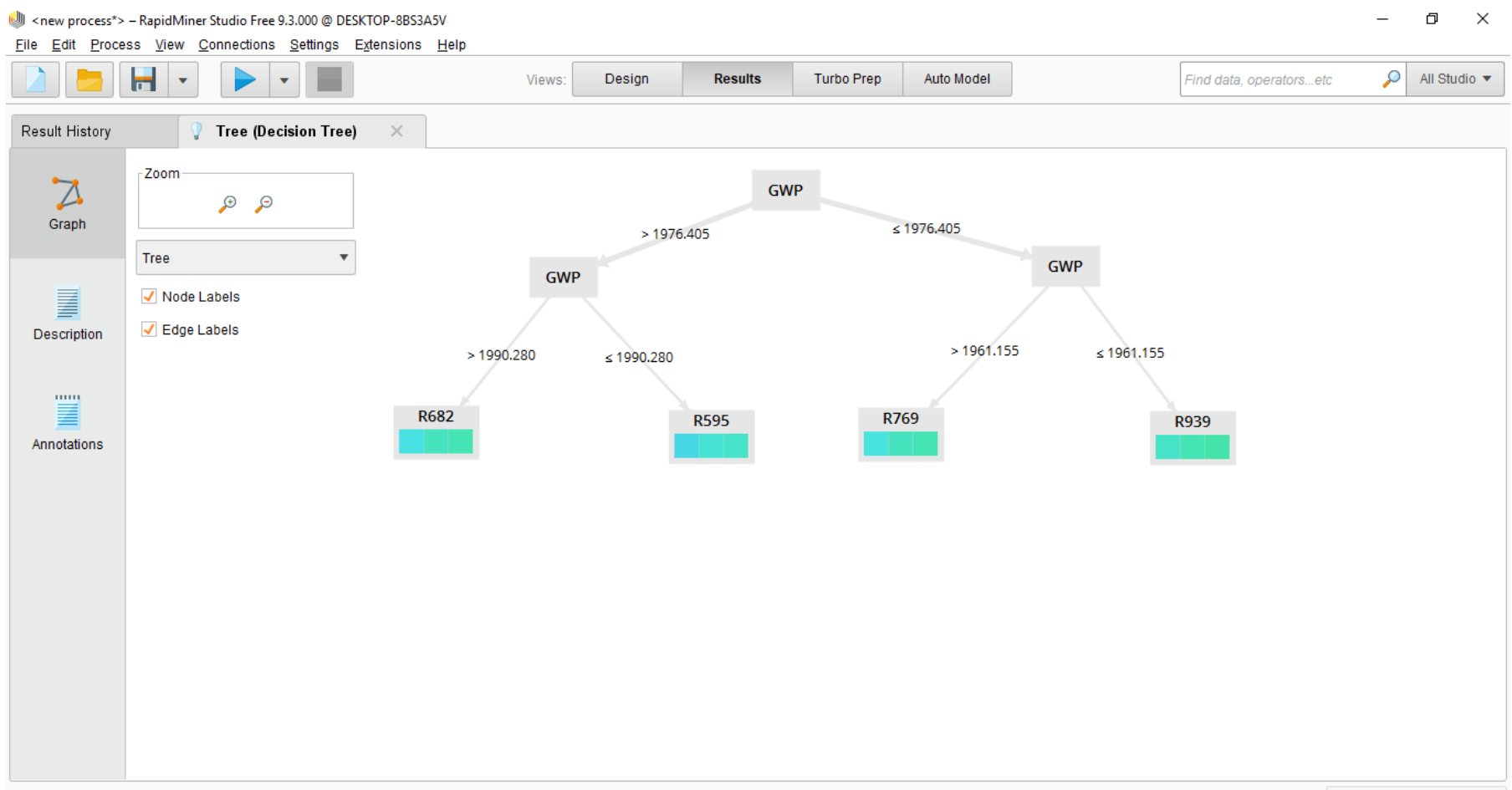

Fig.11. The result of 4 refrigerant that follow refrigerant requirement 


\section{Table 14}

The4 refrigerant result by decision tree in rapid minor software

\begin{tabular}{cccccccccc}
\hline Refrigerant & R134A & R32 & R125 & R1270 & Summary & GWP & Boiling Point & Class A \\
\hline R-No.682 & 8 & 45 & 46 & 1 & 100 & 1,995 & -47.96 & 1 \\
R-No.595 & 7 & 46 & 46 & 1 & 100 & 1,989 & -48.21 & 1 \\
R-No.769 & 9 & 45 & 45 & 1 & 100 & 1,973 & -47.74 & 1 \\
R-No.939 & 11 & 44 & 44 & 1 & 100 & 1,958 & -47.26 & 1 \\
\hline
\end{tabular}

The simulation system used REFPROP and CYCLE_D-HX software from national institute of standards and technology [19] and define new mixture form result of rapid minor software that can see properties and simulation system follow CAN/ANSI/AHRI540 standard air-conditioning, heating, and refrigeration institute (AHRI) showed in Table 14. The result of R-No.682, R-No.595, R-No.769, and R-No.939 had cooling capacity, refrigerant effect, GWP and boiling point were lower than R404A but work and pressure for medium temperature refrigeration system of all R-No.682, R-No.595, RNo.769, and R-No.939 refrigerant was lower than R404A.

Table 14

The simulation system used REFPROP and CYCLE_D-HX

\begin{tabular}{lccccc}
\hline Condition & \multicolumn{5}{c}{ Medium temperature } \\
\hline Refrigerant & R404A & R-No.682 & R-No.595 & R-No.769 & R-No.939 \\
Qevap kJ/kg & 139.02 & 186.88 & 187.76 & 187.66 & 187.55 \\
Qcond kJ/kg & 198.57 & 270.31 & 271.52 & 271.5 & 271.45 \\
Work kJ/kg & 59.55 & 83.43 & 83.76 & 83.84 & 83.9 \\
COPc & 2.335 & 2.24 & 2.242 & 2.238 & 2.235 \\
Pressure at Tev $-6.5^{\circ} \mathrm{C}$ (Psia) & 69.23 & 85.95 & 86.98 & 85.09 & 83.24 \\
Pressure at Tcd $43.5^{\circ} \mathrm{C}$ (Psia) & 331.28 & 422.87 & 425.77 & 420.68 & 415.61 \\
\hline
\end{tabular}

\section{Conclusions}

The artificial intelligence (Al) by data mining technic can predictive environmentally-friendly and energy efficient refrigerant for medium temperature refrigeration. The result of refrigerant mixed by R134A, R32, R125 and R1270 and is consistent with the evolution of the fourth-generation refrigerants that contain a mixture of HFCs, HFOs, HCs, and natural refrigerants, which are required to produce a low-GWP, zero-ODP, high-capacity, low-operating-pressure, and nontoxic refrigerant. In the future, researchers should incorporate R744 in order to use natural refrigerants that are lowcost. The problems of high evaporator pressure and high condenser pressure that impact high refrigerant work can be solved by adjusting the composition of the refrigerant or mix using a refrigerant that operates at low pressure, thereby improving the COP of the refrigerant.

\section{Acknowledgement}

This study was supported by Prince of Songkla University and SANYO S.M.I. (Thailand) Co., Ltd. 


\section{Funding Statement: This research was funded by Prince of Songkla University and SANYO S.M.I. (Thailand) Co., Ltd.}

\section{References}

[1] Kuo, Chung-Feng Jeffrey, Chieh-Hung Lin, and Ming-Hao Lee. "Analyze the energy consumption characteristics and affecting factors of Taiwan's convenience stores-using the big data mining approach." Energy and Buildings 168 (2018): 120-136. https://doi.org/10.1016/i.enbuild.2018.03.021

[2] Saengsikhiao, Piyanut, Juntakan Taweekun, Kittinan Maliwan, Somchai Sae-ung, and Thanansak Theppaya. "Investigation and Analysis of R463A as an Alternative Refrigerant to R404A with Lower Global Warming Potential." Energies 13, no. 6 (2020): 1514. https://doi.org/10.3390/en13061514

[3] Shen, Hanyan, Ke Xu, and James Freihaut. "A statistical study on energy performance of US convenience stores: Investigation of factors and bench marking on store energy use." Energy and Buildings 183 (2019): 792-802. https://doi.org/10.1016/i.enbuild.2018.10.018

[4] Tassou, S. A., Y. Ge, A. Hadawey, and Doug Marriott. "Energy consumption and conservation in food retailing." Applied Thermal Engineering 31, no. 2-3 (2011): 147-156. https://doi.org/10.1016/i.applthermaleng.2010.08.023

[5] Wang, An-Ping, and Pau-Lo Hsu. "The network-based energy management system for convenience stores." Energy and buildings 40, no. 8 (2008): 1437-1445. https://doi.org/10.1016/j.enbuild.2008.01.007

[6] Chou, Ding-chin, Ching-Shan Chang, and Yong-Zhi Hsu. "Investigation and analysis of power consumption in convenience stores in Taiwan." Energy and Buildings 133 (2016): 670-687. https://doi.org/10.1016/i.enbuild.2016.10.010

[7] Evans, J. A., E. C. Hammond, A. J. Gigiel, A. M. Fostera, L. Reinholdt, K. Fikiin, and C. Zilio. "Assessment of methods to reduce the energy consumption of food cold stores." Applied Thermal Engineering 62, no. 2 (2014): 697-705. https://doi.org/10.1016/j.applthermaleng.2013.10.023

[8] Mota-Babiloni, Adrián, Joaquín Navarro-Esbrí, Pavel Makhnatch, and Francisco Molés. "Refrigerant R32 as lower GWP working fluid in residential air conditioning systems in Europe and the USA." Renewable and Sustainable Energy Reviews 80 (2017): 1031-1042. https://doi.org/10.1016/i.rser.2017.05.216

[9] Cardoso, Bruno J., Francisco B. Lamas, Adélio R. Gaspar, and José B. Ribeiro. "Refrigerants used in the Portuguese food industry: Current status." International journal of refrigeration 83 (2017): 60-74. https://doi.org/10.1016/i.ijrefrig.2017.07.013

[10] Sánchez, D., R. Cabello, R. Llopis, J. Catalán-Gil, and L. Nebot-Andrés. "Energy assessment and environmental impact analysis of an R134a/R744 cascade refrigeration plant upgraded with the low-GWP refrigerants R152a, R1234ze (E), propane (R290) and propylene (R1270)." International Journal of Refrigeration 104 (2019): 321-334. https://doi.org/10.1016/j.ijrefrig.2019.05.028

[11] Calleja-Anta, Daniel, Laura Nebot-Andrés, Jesús Catalán-Gil, Daniel Sánchez, Ramón Cabello, and Rodrigo Llopis. "Thermodynamic screening of alternative refrigerants for R290 and R600a." Results in Engineering 5 (2020): 100081. https://doi.org/10.1016/j.rineng.2019.100081

[12] Massuchetto, Luiz Henrique Parolin, Raiza Barcelos Corrêa do Nascimento, Stella Maia Rocha de Carvalho, Hugo Valença de Araújo, and José Vicente Hallak d'Angelo. "Thermodynamic performance evaluation of a cascade refrigeration system with mixed refrigerants: R744/R1270, R744/R717 and R744/RE170." International Journal of Refrigeration 106 (2019): 201-212. https://doi.org/10.1016/j.ijrefrig.2019.07.005

[13] Zhang, Li, Chunguang Yang, Huanying Liu, Ping Du, and Hongyan Gao. "Theoretical Investigation on the properties of R744/R290 mixtures." Procedia Engineering 205 (2017): 1620-1626. https://doi.org/10.1016/i.proeng.2017.10.304

[14] Mečárik, K., and M. Masaryk. "Thermodynamic properties of refrigerants R11, R12, R13, R14, R22, R23, R113, R114, R500 and R502." Heat recovery systems and CHP 11, no. 2-3 (1991): 193-197. https://doi.org/10.1016/0890-4332(91)90135-Q

[15] Bao, Z. Y., D. F. Fletcher, and B. S. Haynes. "Flow boiling heat transfer of Freon R11 and HCFC123 in narrow passages." International Journal of Heat and Mass Transfer 43, no. 18 (2000): 3347-3358.

https://doi.org/10.1016/S0017-9310(99)00379-8 
[16] Chen, Shuangtao, Jionghui Liu, Xiufang Liu, and Yu Hou. "An experimental comparison of heat transfer characteristic between R134-a and R22 in spray cooling." Experimental Thermal and Fluid Science 66 (2015): $206-$ 212. https://doi.org/10.1016/i.expthermflusci.2015.03.015

[17] Fatouh, M., Talaat A. Ibrahim, and A. Mostafa. "Performance assessment of a direct expansion air conditioner working with R407C as an R22 alternative." Applied Thermal Engineering 30, no. 2-3 (2010): 127-133. https://doi.org/10.1016/i.applthermaleng.2009.07.009

[18] Kuczynski, Waldemar, Tadeusz Bohdal, Josua P. Meyer, and Aleksander Denis. "A regressive model for dynamic instabilities during the condensation of R404A and R507 refrigerants." International Journal of Heat and Mass Transfer 141 (2019): 1025-1035. https://doi.org/10.1016/i.ijheatmasstransfer.2019.06.095

[19] Kuczynski, Waldemar. "Experimental research on condensation of R134a and R404A refrigerants in mini-channels during impulsive instabilities. Part I." International Journal of Heat and Mass Transfer 128 (2019): 728-738. https://doi.org/10.1016/j.ijheatmasstransfer.2018.09.045

[20] Kondou, Chieko, Shohei Umemoto, Shigeru Koyama, and Yutaka Mitooka. "Improving the heat dissipation performance of a looped thermosyphon using low-GWP volatile fluids R1234ze (Z) and R1234ze (E) with a superhydrophilic boiling surface." Applied Thermal Engineering 118 (2017): 147-158.

https://doi.org/10.1016/i.applthermaleng.2017.02.073

[21] Colombo, Luigi Pietro Maria, Andrea Lucchini, and Luca Molinaroli. "Experimental analysis of the use of R1234yf and R1234ze (E) as drop-in alternatives of R134a in a water-to-water heat pump." International Journal of Refrigeration (2020). https://doi.org/10.1016/i.ijrefrig.2020.03.004

[22] Sun, Zhili, Qi Cui, Qifan Wang, Jinghong Ning, Jianghe Guo, Baomin Dai, Yongqiang Liu, and Yibo Xu. "Experimental study on CO2/R32 blends in a water-to-water heat pump system." Applied Thermal Engineering 162 (2019): 114303. https://doi.org/10.1016/i.applthermaleng.2019.114303

[23] Hu, Xiaozhen, Tao Yang, Xianyang Meng, and Jiangtao Wu. "Isothermal vapor liquid equilibrium measurements for difluoromethane (R32)+ fluoroethane (R161)+ trans-1, 3, 3, 3-tetrafluoropropene (R1234ze (E)) ternary mixtures." International Journal of Refrigeration 79 (2017): 49-56. https://doi.org/10.1016/j.ijrefrig.2017.04.019

[24] Ju, Fujun, Xiaowei Fan, Yaping Chen, Honglin Zhang, Tuanjie Wang, and Xiangyang Tang. "Performance assessment of heat pump water heaters with R1233zd (E)/HCs binary mixtures." Applied Thermal Engineering 123 (2017): 1345-1355. https://doi.org/10.1016/j.applthermaleng.2017.05.137

[25] Longo, Giovanni A., Simone Mancin, Giulia Righetti, Claudio Zilio, and J. Steven Brown. "Assessment of the lowGWP refrigerants R600a, R1234ze (Z) and R1233zd (E) for heat pump and organic Rankine cycle applications." Applied Thermal Engineering 167 (2020): 114804. https://doi.org/10.1016/i.applthermaleng.2019.114804

[26] Zhu, Jingwei, and Stefan Elbel. "Experimental investigation into the influence of vortex control on transcritical R744 ejector and cycle performance." Applied Thermal Engineering 164 (2020): 114418. https://doi.org/10.1016/i.applthermaleng.2019.114418

[27] Zhang, Yao, Xiaopo Wang, and Jiajia Yin. "Viscosity of saturated mixtures of 1-hexyl-3-methyl-imidazolium bis (trifluoromethylsulfonyl) amide with R600a and R152a." The Journal of Chemical Thermodynamics 141 (2020): 105970. https://doi.org/10.1016/i.jct.2019.105970

[28] Longo, Giovanni A., Giulia Righetti, and Claudio Zilio. "Heat-transfer assessment of the low GWP substitutes for traditional HFC refrigerants." International Journal of Heat and Mass Transfer 139 (2019): 31-38. https://doi.org/10.1016/i.ijheatmasstransfer.2019.04.144

[29] Shaik, Sharmas Vali, and TP Ashok Babu. "Theoretical computation of performance of sustainable energy efficient R22 alternatives for residential air conditioners." Energy Procedia 138 (2017): 710-716. https://doi.org/10.1016/i.egypro.2017.10.205

[30] La Rocca, Vincenzo, and Giuseppe Panno. "Experimental performance evaluation of a vapour compression refrigerating plant when replacing R22 with alternative refrigerants." Applied energy 88, no. 8 (2011): 2809-2815. https://doi.org/10.1016/i.apenergy.2011.01.051

[31] Kasera, Shailendra, and Shishir Chandra Bhaduri. "Performance of R407C as an Alternate to R22: A Review." Energy Procedia 109 (2017): 4-10.

https://doi.org/10.1016/i.egypro.2017.03.032 
[32] Elgendy, E., M. Melike, and M. Fatouh. "Experimental assessment of a split air conditioner working with R-417A under different indoor and outdoor conditions." International Journal of Refrigeration 85 (2018): 268-281. https://doi.org/10.1016/i.ijrefrig.2017.10.005

[33] Fernandez-Seara, Jose, Francisco J. Uhia, Ruben Diz, and J. Alberto Dopazo. "Vapour condensation of R22 retrofit substitutes R417A, R422A and R422D on CuNi turbo C tubes." International journal of refrigeration 33, no. 1 (2010): 148-157. https://doi.org/10.1016/j.ijrefrig.2009.09.006

[34] Aprea, Ciro, and Angelo Maiorino. "An experimental investigation of the global environmental impact of the R22 retrofit with R422D." Energy 36, no. 2 (2011): 1161-1170. https://doi.org/10.1016/i.energy.2010.11.032

[35] Oruc, Vedat, and Atilla G. Devecioğlu. "Thermodynamic performance of air conditioners working with R417A and R424A as alternatives to R22." International journal of refrigeration 55 (2015): 120-128. https://doi.org/10.1016/j.ijrefrig.2015.03.021

[36] Chen, Xiaoning, Cichong Liu, Jingye Yang, and Jiangping Chen. "Experimental study on R-22, R-427A, R-161 and R290 in air-source heat pump for space heating at low ambient temperatures." International Journal of Refrigeration 96 (2018): 147-154. https://doi.org/10.1016/i.ijrefrig.2018.08.021

[37] Devecioğlu, Atilla G., and Vedat Oruç. "The influence of plate-type heat exchanger on energy efficiency and environmental effects of the air-conditioners using R453A as a substitute for R22." Applied Thermal Engineering 112 (2017): 1364-1372. https://doi.org/10.1016/i.applthermaleng.2016.10.180

[38] Yang, Meng, Hua Zhang, Zhaofeng Meng, and Yanbin Qin. "Experimental study on R1234yf/R134a mixture (R513A) as R134a replacement in a domestic refrigerator." Applied Thermal Engineering 146 (2019): 540-547. https://doi.org/10.1016/i.applthermaleng.2018.09.122

[39] Makhnatch, Pavel, Adrián Mota-Babiloni, Alejandro López-Belchí, and Rahmatollah Khodabandeh. "R450A and R513A as lower GWP mixtures for high ambient temperature countries: Experimental comparison with R134a." Energy 166 (2019): 223-235. https://doi.org/10.1016/i.energy.2018.09.001

[40] Heredia-Aricapa, Y., J. M. Belman-Flores, Adrián Mota-Babiloni, Juan Serrano-Arellano, and Juan J. García-Pabón. "Overview of low GWP mixtures for the replacement of HFC refrigerants: R134a, R404A and R410A." International Journal of Refrigeration 111 (2020): 113-123. https://doi.org/10.1016/j.ijrefrig.2019.11.012

[41] Yousif, Qais Abid, Normah Mohd-Ghazali, Agus Sunjarianto Pamitran, and Yushazaziah Mohd-Yunos. "Optimized Condition for Pairing of Different Friction Factor and Viscosity Equations for the Frictional Pressure Drop of R22 and R290." International Journal of Air-Conditioning and Refrigeration (2019): 1950037. https://doi.org/10.1142/S2010132519500378

[42] Zhang, Xiaoyan. "Heat transfer and enhancement analyses of flow boiling for R417A and R22." Experimental thermal and fluid science 35, no. 7 (2011): 1334-1342.

https://doi.org/10.1016/i.expthermflusci.2011.04.020

[43] Llopis, R., E. Torrella, R. Cabello, and D. Sánchez. "HCFC-22 replacement with drop-in and retrofit HFC refrigerants in a two-stage refrigeration plant for low temperature." International journal of refrigeration 35, no. 4 (2012): 810816.

https://doi.org/10.1016/j.ijrefrig.2012.01.001

[44] Oruç, Vedat, Atilla G. Devecioğlu, Uğur Berk, and Ibrahim Vural. "Experimental comparison of the energy parameters of HFCs used as alternatives to HCFC-22 in split type air conditioners." International Journal of Refrigeration 63 (2016): 125-132. https://doi.org/10.1016/j.ijrefrig.2015.11.004

[45] Wang, Fang, Fengkun Wang, Xiaowei Fan, and Zhiwei Lian. "Experimental study on an inverter heat pump with HFC125 operating near the refrigerant critical point." Applied thermal engineering 39 (2012): 1-7. https://doi.org/10.1016/i.applthermaleng.2012.01.016

[46] Arora, Akhilesh, and H. L. Sachdev. "Thermodynamic analysis of R422 series refrigerants as alternative refrigerants to HCFC22 in a vapour compression refrigeration system." International journal of energy research 33, no. 8 (2009): 753-765. https://doi.org/10.1002/er.1508

[47] Saeed, Mian Umar, Shafiq R. Qureshi, Khurram J. Hashmi, Muhammad A. Khan, and Syed N. Danish. "Performance assessment of alternate refrigerants for retrofitting R22 based air conditioning system." Thermal Science 22, no. 2 (2018): 931-941. 


\section{https://doi.org/10.2298/TSCl160811315S}

[48] Devecioglu, Atilla G., and Vedat Oruc. "THE EXPERIMENTAL COMPARISON OF ENVIRONMENTAL IMPACTS OF SOME HFC'S USED INSTEAD OF HCFC-22." ISI BILIMI VE TEKNIGI DERGISI-JOURNAL OF THERMAL SCIENCE AND TECHNOLOGY 36, no. 1 (2016): 99-105.

[49] Paradeshi, Lokesh, M. Srinivas, and S. Jayaraj. "Performance studies of R433A in a direct expansion solar-assisted heat pump." International Journal of Ambient Energy 41, no. 3 (2020): 262-273. https://doi.org/10.1080/01430750.2018.1451369

[50] Subiantoro, A., K. T. Ooi, and A. Z. Junaidi. "Performance and suitabilitycomparisons of some R22 possible substitute refrigerants." In 8thInternational Conference on Compressors and their Systems, pp. 67-76. 2013. https://doi.org/10.1533/9781782421702.2.67

[51] Hari Sankar, R.; Basnth, S.; Ajay Ghosh, K. J.; Sivan, M.; Vyshak Dileep, K., Research on environment friendly alternatives for R22, R12 and R409A refrigerants. International Journal of Recent Technology and Engineering 2019, 8 (1 Special Issue 4), 265-271.

[52] Oruç, Vedat, Atilla G. Devecioğlu, and Sertaç Ender. "Improvement of energy parameters using R442A and R453A in a refrigeration system operating with R404A." Applied Thermal Engineering 129 (2018): 243-249. https://doi.org/10.1016/j.applthermaleng.2017.10.035

[53] Ma, Zhenxi, Fengguo Liu, Changfei Tian, Leilei Jia, and Wei Wu. "Experimental comparisons on a gas engine heat pump using R134a and low-GWP refrigerant R152a." International Journal of Refrigeration (2020). https://doi.org/10.1016/i.ijrefrig.2020.03.007

[54] Jacob, Tabeel A., Ethan P. Matty, and Brian M. Fronk. "Experimental investigation of in-tube condensation of low GWP refrigerant R450A using a fiber optic distributed temperature sensor." International Journal of Refrigeration 103 (2019): 274-286. https://doi.org/10.1016/i.ijrefrig.2019.04.021

[55] Sun, Jian, Wenhua Li, and Borui Cui. "Energy and exergy analyses of R513a as a R134a drop-in replacement in a vapor compression refrigeration system." International Journal of Refrigeration 112 (2020): 348-356. https://doi.org/10.1016/j.ijrefrig.2019.12.014

[56] Mota-Babiloni, Adrián, Joaquín Navarro-Esbrí, Bernardo Peris, Francisco Molés, and Gumersindo Verdú. "Experimental evaluation of R448A as R404A lower-GWP alternative in refrigeration systems." Energy Conversion and Management 105 (2015): 756-762.

https://doi.org/10.1016/i.enconman.2015.08.034

[57] Hu, Xinhao, Zhongbin Zhang, Yuchen Yao, and Qing Wang. "Non-azeotropic refrigerant charge optimization for cold storage unit based on year-round performance evaluation." Applied Thermal Engineering 139 (2018): 395 401. https://doi.org/10.1016/j.applthermaleng.2018.04.089

[58] Bortolini, Marco, Mauro Gamberi, Rita Gamberini, Alessandro Graziani, Francesco Lolli, and Alberto Regattieri. "Retrofitting of R404a commercial refrigeration systems using R410a and R407f refrigerants." International journal of refrigeration 55 (2015): 142-152. https://doi.org/10.1016/i.ijrefrig.2015.02.015

[59] Mota-Babiloni, Adrián, Pavel Makhnatch, and Rahmatollah Khodabandeh. "Recent investigations in HFCs substitution with lower GWP synthetic alternatives: Focus on energetic performance and environmental impact." International Journal of Refrigeration 82 (2017): 288-301. https://doi.org/10.1016/i.ijrefrig.2017.06.026

[60] Mancin, Simone, Davide Del Col, and Luisa Rossetto. "Partial condensation of R407C and R410A refrigerants inside a plate heat exchanger." Experimental thermal and fluid science 36 (2012): 149-157. https://doi.org/10.1016/i.expthermflusci.2011.09.007

[61] Mendoza-Miranda, Juan Manuel, Adrián Mota-Babiloni, and Joaquín Navarro-Esbrí. "Evaluation of R448A and R450A as low-GWP alternatives for R404A and R134a using a micro-fin tube evaporator model." Applied Thermal Engineering 98 (2016): 330-339. https://doi.org/10.1016/j.applthermaleng.2015.12.064

[62] Makhnatch, Pavel, Adrián Mota-Babiloni, Jörgen Rogstam, and Rahmatollah Khodabandeh. "Retrofit of lower GWP alternative R449A into an existing R404A indirect supermarket refrigeration system." International Journal of Refrigeration 76 (2017): 184-192. https://doi.org/10.1016/i.ijrefrig.2017.02.009

[63] Górny, Kasper, Arkadiusz Stachowiak, Przemysław Tyczewski, and Wiesław Zwierzycki. "Lubricity of selected oils in mixtures with the refrigerants R452A, R404A, and R600a." Tribology International 134 (2019): 50-59. https://doi.org/10.1016/i.triboint.2018.12.033 
[64] Devecioğlu, Atilla G., and Vedat Oruç. "An analysis on the comparison of low-GWP refrigerants to alternatively use in mobile air-conditioning systems." Thermal Science and Engineering Progress 1 (2017): 1-5. https://doi.org/10.1016/i.tsep.2017.02.002

[65] Mota-Babiloni, Adrián, Jorge Haro-Ortuno, Joaquín Navarro-Esbrí, and Ángel Barragán-Cervera. "Experimental drop-in replacement of R404A for warm countries using the low GWP mixtures R454C and R455A." International Journal of Refrigeration 91 (2018): 136-145. https://doi.org/10.1016/j.ijrefrig.2018.05.018

[66] Wang, Dong, Yaru Liu, Zunli Kou, Lifang Yao, Yuehong Lu, Leren Tao, and Peng Xia. "Energy and exergy analysis of an air-source heat pump water heater system using CO2/R170 mixture as an azeotropy refrigerant for sustainable development." International Journal of Refrigeration 106 (2019): 628-638. https://doi.org/10.1016/i.ijrefrig.2019.03.007

[67] Fajar, TK Berkah, P. Restu Bagas, S. Ukhi, M. I. Alhamid, and Arnas Lubis. "Energy and exergy analysis of an R410A small vapor compression system retrofitted with R290." Case Studies in Thermal Engineering (2020): 100671. https://doi.org/10.1016/j.csite.2020.100671

[68] Yang, Lixiang, Maoqiong Gong, Hao Guo, Xueqiang Dong, Jun Shen, and Jianfeng Wu. "Isothermal (vapour+ liquid) equilibrium measurements and correlation for the $\{n$-butane (R600)+ 1, 1, 1, 3, 3-pentafluoropropane (R245fa)\} system at temperatures from (303.150 to 373.150) K." The Journal of Chemical Thermodynamics 95 (2016): 49-53. https://doi.org/10.1016/i.jct.2015.11.021

[69] Qiu, Jinyou, and Hua Zhang. "Experimental investigation on two-phase frictional pressure drop of R600a and R600a/3GS oil mixture in a smooth horizontal tube." International Journal of Refrigeration (2020). https://doi.org/10.1016/j.ijrefrig.2020.04.025

[70] Pratama, Felix, Nadhilah Reyseliani, Ahmad Syauqi, Yunus Daud, Widodo Wahyu Purwanto, Praswasti PDK Wulan, and Akhmad Hidayatno. "Thermoeconomic assessment and optimization of wells to flash-binary cycle using pure R601 and zeotropic mixtures in the Sibayak geothermal field." Geothermics 85 (2020): 101778.

https://doi.org/10.1016/i.geothermics.2019.101778

[71] Li, Jian, Qiang Liu, Yuanyuan Duan, and Zhen Yang. "Performance analysis of organic Rankine cycles using R600/R601a mixtures with liquid-separated condensation." Applied Energy 190 (2017): 376-389. https://doi.org/10.1016/i.apenergy.2016.12.131

[72] Wu, Xi, Zhao Yang, Xiaoming Wang, and Yulong Lin. "Experimental and theoretical study on the influence of temperature and humidity on the flammability limits of ethylene (R1150)." Energy 52 (2013): 185-191. https://doi.org/10.1016/j.energy.2013.01.042

[73] Zhang, Yeqiang, Yongning He, Yanling Wang, Xuehong Wu, Mingzheng Jia, and Yi Gong. "Experimental investigation of the performance of an R1270/CO2 cascade refrigerant system." International Journal of Refrigeration (2020). https://doi.org/10.1016/j.ijrefrig.2020.02.017

[74] CAN/ANSI/AHRI540, Performance Rating of Positive Displacement Refrigerant Compressors and Compressor Units Air-Conditioning, Heating, and Refrigeration Institute (AHRI) (E) 2015, 5.

https://doi.org/10.1016/j.apenergy.2011.06.049

[75] Aprea, Ciro, Angelo Maiorino, and Rita Mastrullo. "Change in energy performance as a result of a R422D retrofit: An experimental analysis for a vapor compression refrigeration plant for a walk-in cooler." Applied energy 88, no. 12 (2011): 4742-4748. https://doi.org/10.1016/i.ijrefrig.2017.11.009

[76] Lumpkin, Domenique R., Ammar M. Bahman, and Eckhard A. Groll. "Two-phase injected and vapor-injected compression: Experimental results and mapping correlation for a R-407C scroll compressor." International Journal of Refrigeration 86 (2018): 449-462.

https://doi.org/10.1016/i.ijrefrig.2017.11.009

[77] Lausch, Angela, Andreas Schmidt, and Lutz Tischendorf. "Data mining and linked open data-New perspectives for data analysis in environmental research." Ecological Modelling 295 (2015): 5-17. https://doi.org/10.1016/i.ecolmodel.2014.09.018

[78] Bell, Ian H., Piotr A. Domanski, Mark O. McLinden, and Gregory T. Linteris. "The hunt for nonflammable refrigerant blends to replace R-134a." International Journal of Refrigeration 104 (2019): 484-495. https://doi.org/10.1016/j.ijrefrig.2019.05.035

[79] Domanski, Piotr A., Riccardo Brignoli, J. Steven Brown, Andrei F. Kazakov, and Mark O. McLinden. "Low-GWP refrigerants for medium and high-pressure applications." International Journal of Refrigeration 84 (2017): 198209.

https://doi.org/10.1016/i.ijrefrig.2017.08.019 
[80] Brignoli, Riccardo, J. Steven Brown, Harrison M. Skye, and Piotr A. Domanski. "Refrigerant performance evaluation including effects of transport properties and optimized heat exchangers." International Journal of Refrigeration 80 (2017): 52-65.

https://doi.org/10.1016/j.ijrefrig.2017.05.014 